Evidenza di disfunzione endoteliale in pazienti diabetici di tipo 2 con arterie coronariche angiograficamente normali. Studio con tonometria arteriosa digitale

\title{
Endothelial dysfunction in type 2 diabetic patients with normal coronary arteries. A peripheral arterial tonometry study
}

\author{
Donatella Ruggiero, Gianluigi Savarese, Roberto Formisano, Ada Bologna, \\ Giacomo Mattiello, Elisabetta Pirozzi, Francesco Gambardella, Francesco Lo ludice, \\ Laura Petraglia, Alice Vitagliano, Laura Casaretti, Giuseppe Luca Della Ratta, \\ Susanna Mosca, Pasquale Perrone Filardi
}

\begin{abstract}
Endothelial dysfunction in type 2 diabetic patients with normal coronary arteries. A peripheral arterial tonometry study. D. Ruggiero, G. Savarese, R. Formisano, A. Bologna, G. Mattiello, E. Pirozzi, F. Gambardella, F. Lo Iudice, L. Petraglia, A. Vitagliano, L. Casaretti, G.L. Della Ratta, S. Mosca, P. Perrone Filardi.

Aim: to evaluate endothelial function (EF) in diabetic and non-diabetic patients without CAD by peripheral artery tonometry (PAT) technique.

Methods: a cohort of 94 patients (55 men and 39 postmenopausal women; mean age $63 \pm 9$ years) undergoing coronary angiography was divided into 2 groups: 58 patients with DM and (group 1) and 36 patients without DM. Endothelial dysfunction (ED) was assessed by digital pulse amplitude, using a fingertip peripheral arterial tonometry (PAT). As a measure of ED, reactive hyperemia index (RHI)
\end{abstract}

was calculated as the ratio of the digital pulse volume during reactive hyperemia following 5 min ischemia and its basal value.

Results: prevalence of cardiovascular risk factors was similar between the two groups. RHI values were significantly lower in diabetic patients compared to non-diabetics $(1.72 \pm 0.34$ vs $2.00 \pm 0.44 ; p<0.005)$ and they correlated with levels of glycosylated hemoglobin $(p=0.05 ; r=-0.266)$.

Conclusion: despite similar level of other risk factors, EF was much more impaired in diabetic patients than in non-diabetics. These evidences further support the impact of DM on cardiovascular risk.

Keywords: endothelium dysfunction, pulse artery tonometry, diabetes mellitus.

Monaldi Arch Chest Dis 2012; 78: 34-39.

Dipartimento di Medicina Interna, Scienze Cardiovascolari ed Immunologiche, Università degli Studi di Napoli "Federico II".

Corresponding author: Prof. Pasquale Perrone-Filardi; Via Pansini, 5; I-80131 Napoli, Italy; Tel/Fax: +39 0817462232 ; E-mail address:fpperron@unina.it

\section{Introduzione}

La disfunzione endoteliale è un marcatore precoce di aterosclerosi, in grado di predire lo sviluppo di malattia coronarica [1] prima della manifestazione clinica e del riscontro angiografico $[2,3]$. La prolungata e ripetuta esposizione ai fattori di rischio cardiovascolari provoca a livello endoteliale un'alterata produzione e metabolizzazione di ossido nitrico (NO) e la sintesi di reagenti ossidanti dell'ossigeno (ROS). Questi fattori determinano disfunzione e perdita dell'integrità endoteliale e la conseguente evoluzione verso l'aterosclerosi, la trombosi e l'ischemia miocardica [4].

Descritta per la prima volta in soggetti ipertesi [5], la disfunzione endoteliale è stata successivamente riscontrata in pazienti con diabete di tipo 1 [6] e 2 [7], nella malattia coronarica [8-10] e nello scompenso cardiaco [11], anche se al pari di altri indicatori di danno d'organo, resta incerto il valore di target terapeutico [12]. Recenti studi ne hanno dimostrato il ruolo di predittore di eventi cardiovascolari, indipendentemente dalla presenza di lesioni coronariche. [13] Sulla base di queste osservazioni, la valutazione della disfunzione endoteliale è considerata un valido strumento per la stratificazione precoce dei pazienti a rischio di eventi cardiovascolari. Pertanto sono state proposte e sviluppate nuove metodiche in grado di eseguirne un accurato esame in maniera non invasiva, quali la dilatazione flussomediata (flow mediated dilatation, FMD) dell'arteria brachiale e, più recentemente, la tonometria arteriosa periferica (peripheral artery tonometry, PAT).

Negli ultimi anni numerosi studi hanno dimostrato una correlazione significativa tra la risposta vasodilatatoria del circolo coronarico all'iniezione intracardiaca di acetilcolina durante esame coronarografico e FMD [14]. Tale evidenza ha supportato la FMD quale tecnica gold standard per la valutazione non invasiva della funzione endoteliale. Tutta- 
via l'impiego routinario di questa metodica è fortemente limitato dai lunghi tempi d'esecuzione dell'esame e dalla lunga preparazione di cui necessita il medico per divenire un operatore esperto.

Recentemente sono state riportate numerose evidenze di una correlazione tra la valutazione dell'iperemia reattiva del letto vascolare delle dita della mano mediante PAT e studio della funzione endoteliale con metodica invasiva e non invasiva (FMD) $[15,16]$. Inoltre, Rubinstein et al. [17] hanno dimostrato che la disfunzione endoteliale, valutata con metodica PAT, è in grado di predire l'insorgenza tardiva di eventi cardiovascolari in pazienti asintomatici con e senza malattia coronarica. Da queste osservazioni consegue che la valutazione della funzione endoteliale mediante PAT potrebbe rappresentare uno strumento utile nella stratificazione del rischio cardiovascolare.

In alcuni studi è stata riscontrata la presenza di disfunzione endoteliale nelle fasi iniziali del diabete mellito di tipo 2 (DM) [18] e nei parenti di primo grado di pazienti diabetici con ridotta sensibilità all'insulina, ma senza DM manifesto [19]. Tuttavia, solo pochi autori hanno studiato la funzione endoteliale in pazienti con DM senza evidenza di malattia aterosclerotica all'angiografia coronarica $[20,21]$ ed inoltre mancano finora in letteratura dati in questi pazienti ottenuti mediante metodica PAT.

Pertanto, l'obiettivo del nostro studio è stato valutare la funzione endoteliale con metodica PAT nei pazienti diabetici e non diabetici senza evidenza angiografica di malattia coronarica.

\section{Materiali e metodi}

\section{Popolazione}

Sono stati arruolati 94 pazienti (55 uomini e 39 donne in postmenopausa di età media pari a $63 \pm 9$ anni). Nessuna delle donne arruolate nello studio praticava terapia ormonale sostitutiva.

\section{Protocollo di ricerca}

I pazienti sono stati selezionati secondo i seguenti criteri di inclusione: 1) indicazione ad angiografia coronarica (sintomi e/o evidenza di ischemia inducibile al test ergometrico o ad indagini di stress imaging); 2) anamnesi patologica negativa per pregresso infarto del miocardio; 3) assenza di stenosi emodinamicamente significative all'angiografia coronarica; 4) evidenza ecocardiografica di conservata cinesi globale e segmentaria del ventricolo sinistro; 5) rilascio del consenso informato. Sono stati considerati criteri di esclusione la malattia di Raynaud e la presenza di gravi patologie endocrine, epatiche, renali o infiammatorie.

Come previsto dal protocollo, i pazienti hanno effettuato nella stessa giornata valutazione della funzione endoteliale mediante metodica PAT ed esame angiografico. In ciascun paziente è stata valutata la presenza dei seguenti fattori di rischio cardiovascolare: storia familiare di patologia coronarica, sovrappeso (definito dall'indice di massa corporea $[\mathrm{BMI}]>25$ ), ipertensione arteriosa (pressione arteriosa sistolica $>140 \mathrm{mmHg}$, pressione diastolica $>90$ $\mathrm{mmHg}$ o assunzione di farmaci antipertensivi), di- slipidemia (colesterolo totale $>200 \mathrm{mg} / \mathrm{dl}$, o assunzione di farmaci specifici), tabagismo ( $\geq 5 \mathrm{sig} / \mathrm{die}$ nell'ultimo mese), assunzione di farmaci ipoglicemizzanti orali o insulina e farmaci di pertinenza cardiovascolare.

La diagnosi di DM è stata posta secondo le linee guida della Società Americana di Diabetologia [22]. In accordo con le Linee Guida della Società Europea di Cardiologia, la presenza di malattia coronarica è stata definita dalla presenza di stenosi emodinamicamente significativa (evidenza angiografica di almeno 1 stenosi con restringimento del lume coronarico $\geq 70 \%$ coinvolgente almeno 1 vaso coronarico maggiore) [23].

\section{Valutazione della funzione endoteliale}

La PAT (Itamar Medical Ltd., Caesarea, Israele) è una metodica non invasiva che sfrutta la registrazione pletismografica pulsatile del volume delle arterie digitali mediante sonde pneumatiche applicate alle estremità della stessa falange distale di entrambe le mani. La sonda digitale consta di un sensore a forma di ditale che impartisce una pressione uniforme sull'intera superficie della falange distale e misura i cambiamenti pulsatili di volume delle arterie. Tale pressione impedisce la dilatazione venosa distale e quindi la stasi venosa, che potrebbe indurre una risposta riflessa vasocostrittrice veno-arteriosa. La sonda digitale è connessa da un tubo flessibile ad un'unità centrale che determina il gonfiaggio della stessa. Le variazioni di pressione sono poi filtrate, amplificate, visualizzate e archiviate per ulteriori analisi. Ai soggetti arruolati è stato richiesto il digiuno per le 12 ore precedenti il test e l'astensione da fumo, alcool o caffeina. Tutti i farmaci vasoattivi sono stati sospesi almeno 24 ore prima dello studio. La procedura è stata effettuata in una stanza tranquilla e a temperatura controllata, cioè compresa tra 21 e $24^{\circ} \mathrm{C}$; vestiti stretti, orologi, anelli e bracciali sono stati rimossi per evitare che interferissero con il flusso ematico delle braccia e delle dita. Inoltre, prima del test, i pazienti sono rimasti comodamente seduti o sdraiati per almeno 15 minuti, in modo tale da raggiungere uno stato di rilassamento cardiovascolare.

La PAT rileva la risposta iperemica reattiva delle arterie digitali che si sviluppa in seguito all'ischemia indotta da un manicotto di uno sfigmomanometro posto all'estremità prossimale del braccio in esame (braccio di studio), mentre il controlaterale fungeva da controllo (braccio di controllo). Le sonde PAT sono state collocate sullo stesso dito di entrambe le mani per la registrazione continua del segnale. Sono stati registrati un segnale di base per 3-10 minuti, un segnale post-occlusione del flusso di sangue per 4.55.5 minuti (ottenuto dopo gonfiaggio del manicotto ad una pressione $>60 \mathrm{mmHg}$ rispetto alla pressone sistolica basale, e in ogni caso raggiungendo una pressione sistolica $>200 \mathrm{mmHg}$ ), e un segnale di 35 minuti dopo il rilascio del manicotto. I dati ottenuti sono stati analizzati da un computer e quindi in modalità operatore-indipendente [15, 24].

L'entità della risposta iperemica è espressa dall'Indice di Iperemia Reattiva (Reactive Hyperemia Index, RHI), ossia il rapporto tra l'ampiezza media del segnale PAT registrato in un intervallo di tempo 
di un minuto a partire da un minuto dopo lo sgonfiaggio del manicotto e l'ampiezza media del segnale PAT registrato nei 3 minuti e 30 secondi che precedono il gonfiaggio del manicotto. Il valore dell'RHI ottenuto al braccio in esame è stato normalizzato confrontandolo al braccio di controllo (Figura 1) $[15,16]$. È stato assunto quale valore normale di riferimento, un RHI pari a 1.68 calcolato come valore medio $(2.34 \pm 0.33)$ meno 2 deviazioni standard in una popolazione di 20 soggetti sani, senza alcun fattore di rischio cardiovascolare.

\section{Analisi statistica}

L'analisi statistica è stata eseguita utilizzando SPSS Statistical Software 14.0 (SPSS Inc., Chicago, Illinois). I dati sono stati espressi come media \pm 1 SD per le variabili continue e come frequenza per le variabili categoriche. Il test T-Student è stato utilizzato per paragonare i valori di RHI tra il gruppo dei pazienti diabetici e quello dei non diabetici, entrambi non coronaropatici. L'omogeneità dei due gruppi di studio per quanto riguarda i fattori di rischio cardiovascolare e la terapia farmacologica assunta è stata valutata mediante test T-Student per le variabili continue e test ChiSquare per le variabile dicotomiche.

\section{Risultati}

I pazienti arruolati nello studio sono stati suddivisi in due gruppi in base alla diagnosi di DM: pazienti con DM (gruppo 1, $\mathrm{n}=58)$, pazienti senza DM (gruppo 2, $n=36$ ).

I due gruppi risultavano omogeni per quanto riguarda le caratteristiche demografiche ed i fattori di rischio cardiovascolare (Tabella 1).

L'RHI è risultato significativamente più basso nei pazienti diabetici rispetto ai pazienti non diabetici (rispettivamente $1.72 \pm 0.34$ vs $2.00 \pm 0.44$; $\mathrm{p}<0.005$; Figura 2). Risultati analoghi sono stati ottenuti esprimendo l'RHI come logaritmo naturale (L_RHI)(rispettivamente $0.5 \overline{3} \pm 0.20$ vs $0.67 \pm 0.21 ; \mathrm{p}<0.008)$. I livelli di emoglobina glicata sono risultati $7.20 \pm 0.95 \%$ e sono correlati inversamente ai valori di RHI $(\mathrm{p}=0.05, \mathrm{r}=-0.266) \quad(\mathrm{Fi}-$ gura 3$)$.

\section{Discussione}

I risultati di questo studio dimostrano che la funzione endoteliale, valutata mediante me- todica PAT, è severamente compromessa nei pazienti diabetici rispetto ai pazienti non diabetici anche in assenza di lesioni coronariche angiograficamente significative.

Simili risultati sono stati ottenuti da Nitenberg et al. [20] che hanno dimostrato la presenza di disfunzione endoteliale valutata con metodica invasiva, in una coorte di 11 pazienti diabetici con esame coronarografico negativo rispetto a 7 soggetti sani di controllo. Tuttavia in questo studio i pazienti diabetici erano paragonati a soggetti sani e quindi i due gruppi di studio non risultavano omogeni per caratteristiche demografiche e distribuzione dei fattori di rischio cardiovascolare, variabili che potrebbero aver contribuito a determinare una significativa differenza di funzione endoteliale nel gruppo dei pazienti diabetici. Viceversa, siccome nel nostro studio sia i pazienti diabetici che i non diabetici risultavano appaiati per caratteristiche demografiche e fattori di
Tabella 1. - Caratteristiche cliniche dei gruppi di studio

\begin{tabular}{|c|c|c|c|}
\hline & $\begin{array}{l}\text { DM+ } \\
\text { n. } 58\end{array}$ & $\begin{array}{l}\text { DM } \\
\text { n. } 36\end{array}$ & $\mathbf{p}$ \\
\hline $\begin{array}{l}\text { Familiarità per coronaropatia } \\
\text { (\% dei pazienti) }\end{array}$ & $\begin{array}{c}13 \\
(22.42 \%)\end{array}$ & $\begin{array}{c}4 \\
(11.11 \%)\end{array}$ & 0.270 \\
\hline Età (anni) & $62.96 \pm 8.82$ & $63.50 \pm 9.63$ & 0.768 \\
\hline $\begin{array}{l}\text { Sesso maschile } \\
\text { (\% dei pazienti) }\end{array}$ & $\begin{array}{c}33 \\
(56.89 \%)\end{array}$ & $\begin{array}{c}22 \\
(61.11 \%)\end{array}$ & 0.525 \\
\hline $\begin{array}{l}\text { Ipertensione arteriosa } \\
\text { (\% dei pazienti) }\end{array}$ & $\begin{array}{c}43 \\
(74.14 \%)\end{array}$ & $\begin{array}{c}29 \\
(80.55 \%)\end{array}$ & 0.618 \\
\hline $\begin{array}{l}\text { Pressione arteriosa } \\
\text { sistolica }(\mathrm{mmHg})\end{array}$ & $130.16 \pm 12.72$ & $126.90 \pm 10.64$ & 0.197 \\
\hline $\begin{array}{l}\text { Pressione arteriosa } \\
\text { diastolica }(\mathrm{mmHg})\end{array}$ & $76.06 \pm 8.59$ & $77.76 \pm 9.02$ & 0.455 \\
\hline Colesterolo Totale $(\mathrm{mg} / \mathrm{dl})$ & $175.44 \pm 35.56$ & $188.20 \pm 45.88$ & 0.085 \\
\hline $\begin{array}{l}\text { Fumo } \\
\text { (\% dei pazienti) }\end{array}$ & $\begin{array}{c}26 \\
(44.82 \%)\end{array}$ & $\begin{array}{c}16 \\
(44.44 \%)\end{array}$ & 1.000 \\
\hline BMI $\left(\mathrm{Kg} / \mathrm{m}^{2}\right)$ & $27 \pm 4$ & $26 \pm 3$ & 0.245 \\
\hline Emoglobina glicata (\%) & $7.20 \pm 0.95$ & 1 & 1 \\
\hline $\begin{array}{l}\text { Statine } \\
\text { (\%dei pazienti) }\end{array}$ & $\begin{array}{c}35 \\
(60.34 \%)\end{array}$ & $\begin{array}{c}21 \\
(58.33 \%)\end{array}$ & 1.000 \\
\hline $\begin{array}{l}\text { Antiaggreganti } \\
\text { (\% dei pazienti) }\end{array}$ & $\begin{array}{c}31 \\
(53.45 \%)\end{array}$ & $\begin{array}{c}19 \\
(52.77 \%)\end{array}$ & 1.000 \\
\hline $\begin{array}{l}\text { Antagonisti recettori AT1 } \\
\text { (\% dei pazienti) }\end{array}$ & $\begin{array}{c}22 \\
(37.93 \%)\end{array}$ & $\begin{array}{c}5 \\
(13.88 \%)\end{array}$ & 0.018 \\
\hline $\begin{array}{l}\text { ACE inibitori } \\
\text { (\% dei pazienti) }\end{array}$ & $\begin{array}{c}16 \\
(27.58 \%)\end{array}$ & $\begin{array}{c}14 \\
(38.88 \%)\end{array}$ & 0.265 \\
\hline $\begin{array}{l}\text { Calcio antagonisti } \\
\text { (\% dei pazienti) }\end{array}$ & $\begin{array}{c}14 \\
(24.14 \%)\end{array}$ & $\begin{array}{c}3 \\
(8.3 \%)\end{array}$ & 0.060 \\
\hline $\begin{array}{l}\beta \text {-bloccanti } \\
\text { (\% dei pazienti) }\end{array}$ & $\begin{array}{c}8 \\
(4.37 \%)\end{array}$ & $\begin{array}{c}21 \\
(11.02 \%)\end{array}$ & 0.000 \\
\hline
\end{tabular}

DM: diabete mellito

BMI: indice di massa corporea

ACE: enzima convertitore angiotensina 


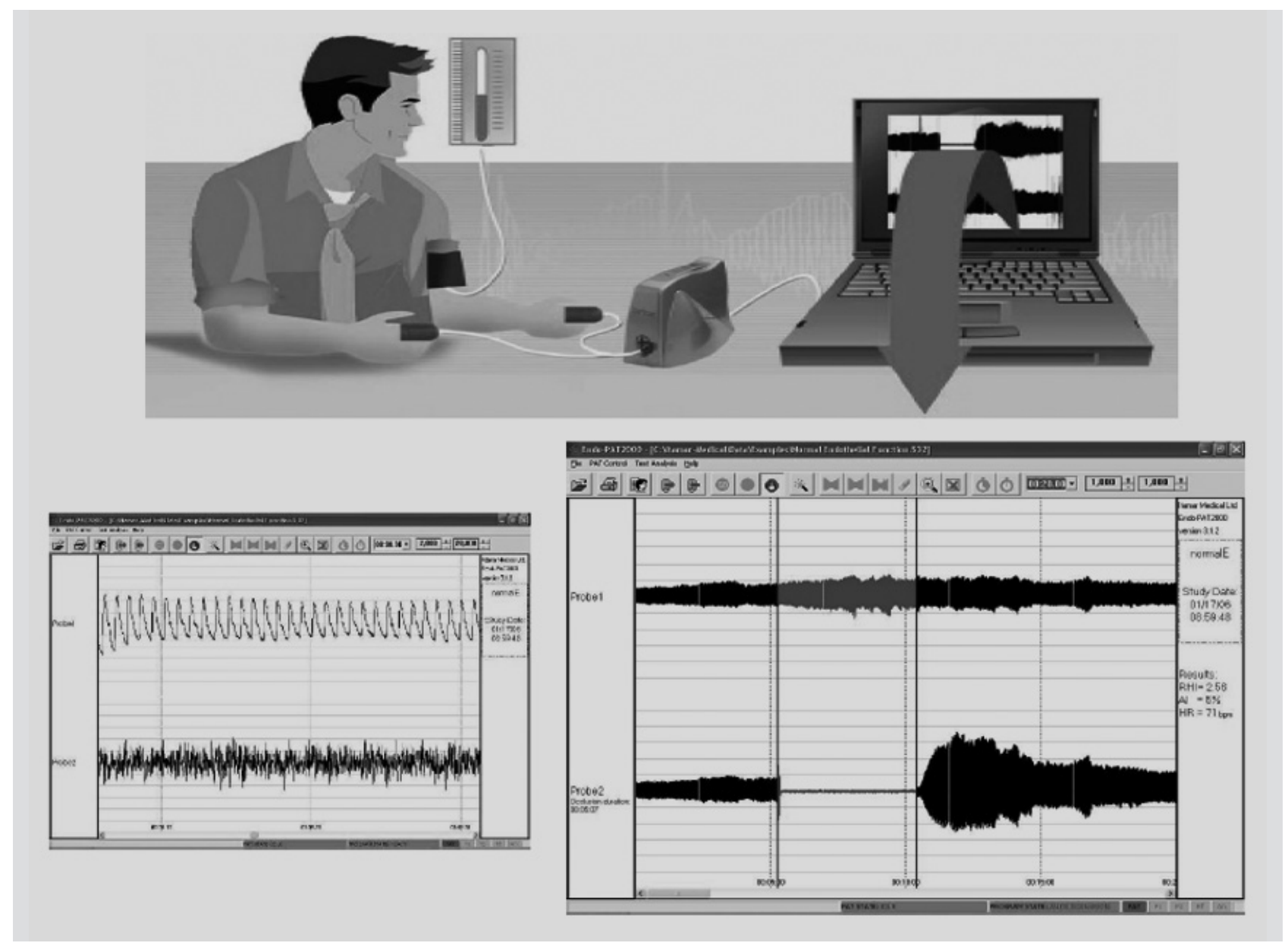

Figura 1. Schema riassuntivo del funzionamento della tonometria arteriosa periferica (PAT)(Itamar Medical Ltd., Caesarea, Israele).

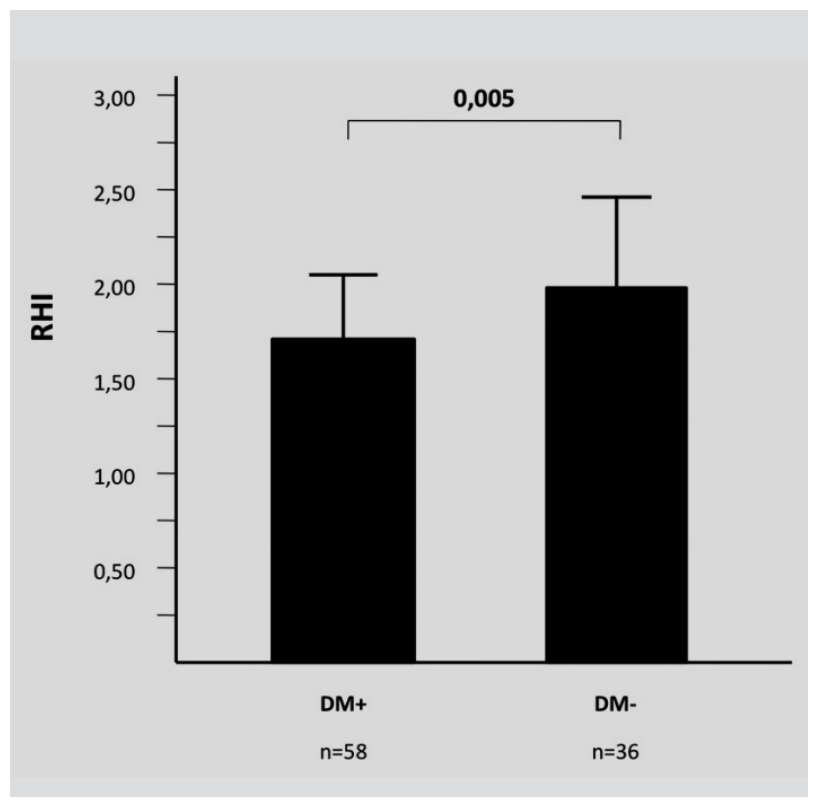

Figura 2. Indice di iperemia reattiva (RHI) nei gruppi di studio.

rischio cardiovascolari, la significativa differenza di funzione endoteliale rilevabile nei due gruppi non può essere giustificata dalla presenza di ulteriori fattori di rischio cardiovascolari concomitanti che frequentemente si associano al DM. Ciò rappresenta una ulteriore conferma del ruolo patogenetico che il DM riveste nel determinare l'insorgenza di disfun- zione endoteliale, anche in assenza di patologia coronarica conclamata.

Numerosi sono gli studi che hanno indagato i meccanismi fisiopatologici con i quali il DM determina disfunzione endoteliale. Cosentino et al. [25] hanno osservato un aumento dei livelli di mRNA e dell'espressione dell'eNOS in cellule endoteliali di aorta umana esposte a prolungate alte concentrazioni di glucosio. A ciò conseguiva un aumento del $40 \%$ della sintesi di NO, ma soprattutto un incremento del $300 \%$ della produzione di $\mathrm{O}_{2}{ }^{-}$. L'interazione tra queste due molecole determinava l'inattivazione del NO e la produzione di perossinitrito, con il conseguente sviluppo di danno endoteliale.

Il ruolo dell'iperglicemia nell'insorgenza della disfunzione endoteliale è stato esplorato anche da Makimattila et al. [26] che hanno riportato una correlazione tra controllo glicemico e funzione endoteliale coronarica in 18 pazienti insulino-dipendenti.

Nel presente studio è stata utilizzata la metodica PAT per la valutazione della funzione endoteliale. Tale innovativa metodica è già stata utilizzata in pazienti non coronaropatici in precedenti studi. Bonetti et al. [15] hanno valutato la funzione endoteliale sia invasivamente con il test all'acetlilcolina, sia non invasivamente con tecnica PAT, dimostrando una riduzione significativa del valore di RHI nei pazienti con disfunzione endoteliale coronarica evidenziata al test invasivo durante angiografia. Anche Kuvin et al. [16] hanno confrontato le metodiche FMD e PAT, dimostrando che a ridotti valori di FMD corrispon- 


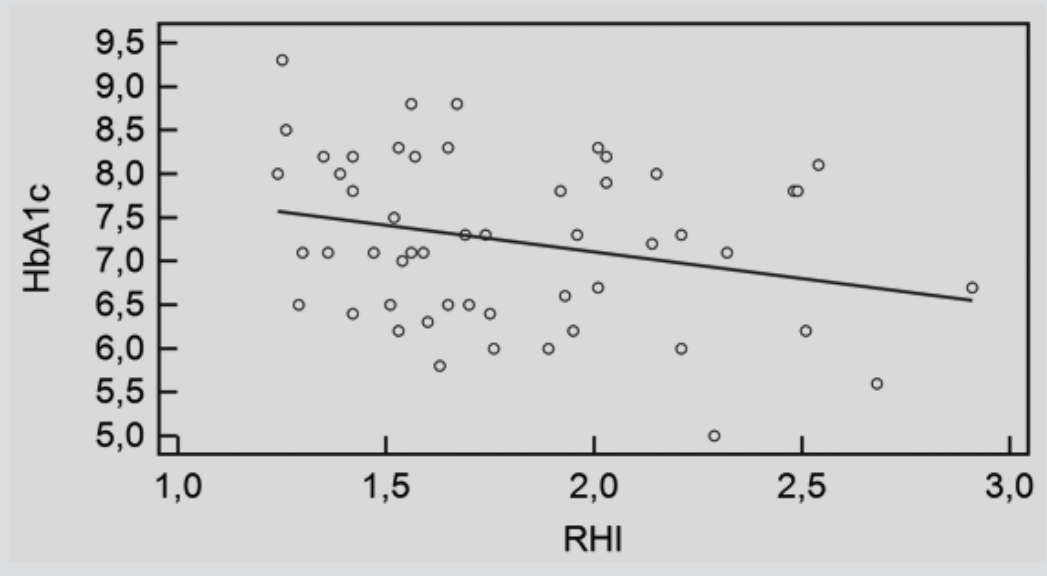

Figura 3. Regressione lineare tra indice di iperemia reattiva (RHI) ed emoglobina glicata (HbA1c).
Conclusioni

Nei pazienti con DM di tipo 2 , la funzione endoteliale risulta maggiormente compromessa rispetto ai pazienti non diabetici di controllo a parità di altri fattori di rischio. Ciò contribuisce a spiegare l'impatto fisiopatogenetico del DM sullo sviluppo dell'aterosclerosi coronarica e delle sue complicanze.

Lo studio dimostra come la metodica PAT sia un valido strumento per la identificazione precoce della disfunzione endoteliale di potenziale impiego in larghe popolazioni di soggetti a rischio cardiovascolare.

\section{Riassunto}

devano equivalenti riduzioni di RHI. Da questi studi si evince che le metodiche PAT e FMD correlano strettamente con la valutazione invasiva della funzione endoteliale coronarica, pur essendo la PAT di esecuzione più semplice e meno operatore-dipendente della FMD.

Infine, è stato di recente esplorato il ruolo prognostico della disfunzione endoteliale valutata con PAT. Infatti Rubinshtein et al. [17] hanno riportato in 270 pazienti sottoposti ad un follow-up mediano di 5.8 anni una più alta incidenza di eventi avversi (morte cardiovascolare, infarto del miocardio, rivascolarizzazione ed ospedalizzazione per patologie cardiovascolari) nei pazienti con disfunzione endoteliale, definita dal logaritmo naturale di RHI (L_RHI)<0.4. L'endpoint composito non soltanto si verificava più frequentemente durante il follow-up nei pazienti con L_RHI più basso rispetto a quelli con L_RHI più alto, ma era anche capace di predire futuri eventi avversi.

I risultati dello studio supportano dunque l'utilità della metodica PAT nella valutazione non invasiva della funzione endoteliale e forniscono informazioni di potenziale utilità per la gestione clinica dei pazienti ad alto rischio cardiovascolare.

\section{Limiti dello studio}

Una limitazione di questo studio è rappresentata dalla mancanza di un follow-up. Infatti in letteratura sono disponibili pochi dati per valutare l'impatto prognostico della disfunzione endoteliale valutata con metodica PAT, sugli outcome cardiovascolari. Questi dati potrebbero essere utili per testare l'efficacia di strategie terapeutiche più aggressive in pazienti diabetici con disfunzione endoteliale ma senza CAD.

Inoltre nello studio molti pazienti utilizzavano farmaci che possono influenzare la funzione endoteliale. Sebbene questi siano stati sospesi almeno 24 ore prima della misurazione dell'RHI, non si può escludere, ad eccezione che per i nitrati, un effetto vasoattivo persistente in grado di influenzare i risultati del test. Tuttavia, dal momento che questi farmaci hanno dimostrato migliorare la funzione endoteliale, i risultati potrebbero essere semplicemente sottostimati.
Obiettivo: scopo dello studio è valutare la funzione endoteliale mediante tonometria arteriosa periferica (PAT) in pazienti senza malattia aterosclerotica coronarica con e senza diabete mellito $(D M)$.

Metodi: sono stati arruolati 94 pazienti (55 иomini e 39 donne in postmenopausa di età media pari a $63 \pm 9$ anni) sottoposti ad angiografia coronarica e sono stati suddivisi in due gruppi in base alla diagnosi di DM: pazienti con DM (gruppo $1, n=58$ ), pazienti senza DM (gruppo 2, $n=36$ ). La disfunzione endoteliale è stata valutata mediante registrazioni pulsatili del volume delle arterie, utilizzando la tonometria arteriosa periferica (PAT). Come misura di ED è stato calcolato l'RHI (Reactive Hyperemia Index), definito come rapporto tra il volume presente al tono arterioso nel dito in condizioni di ischemia e lo stesso volume in condizioni basali.

Risultati: la prevalenza dei fattori di rischio cardiovascolare risultava simile nei 2 gruppi di studio. Dai dati ottenuti si rileva un valore medio di RHI significativamente più basso nei pazienti diabetici rispetto ai pazienti non diabetici (rispettivamente $1.72 \pm 0.34$ vs $2.00 \pm 0.44 ; p<0.005$ ). I valori di $R H I$ era correlati inversamente ai livelli di emoglobina glicata $(p=0.05 ; r=-0.266)$.

Conclusione: a parità di fattori di rischio cardiovascolare, i pazienti diabetici presentano una più severa alterazione della funzione endoteliale rispetto ai pazienti non diabetici. Queste evidenze contribuiscono a spiegare patogeneticamente il ruolo del DM quale fattore di rischio cardiovascolare.

Parole chiave: disfunzione endoteliale, tonometria arteriosa periferica, diabete mellito.

\section{ABBREVIAZIONI}

NO: ossido nitrico

ROS: reactive oxygen species - reagenti ossidanti dell'ossigeno FMD: flow mediated dilatation - dilatazione flusso-mediata PAT: peripheral artery tonometry - tonometria arteriosa periferica

DM: diabete mellito

BMI: body mass index - indice di massa corporea

RHI: reactive hyperemia index - indice di iperemia reattiva 


\section{Bibliografia}

1. Kim J, Montagnani M, Koh KK, Quon MJ. Reciprocal Relationship between insulin resistance and endothelial dysfunction: molecular and pathophysiological mechanisms. Circulation 2006; 113: 1888-904.

2. Thorne S, Mullen MJ, Clarkson P et al. Early endothelial dysfunction in adults at risk from atherosclerosis: Different responses to L-arginine. J Am Coll Cardiol 1998; 32: 110-6.

3. Ludmer PL, Selwyn AP, Shook TL et al. Paradoxical vasoconstriction induced by acetylcholine in atherosclerotic coronary arteries. N Engl J Med 1986; 315: 1046-51.

4. Selwyn AP, Kinlay S, Libby P, Ganz P: Atherogenic lipids, vascular dysfunction, and clinical signs of ischemic heart disease. Circulation 1997; 95: 5-7.

5. Panza JA, Quyyumi AA, Brush JE jr, Epstein SE. Abnormal endothelium-dependent vascular relaxationin patients with essential hypertension. $N$ Engl J Med 1990; 323: 22-27.

6. Park JB, Charbonneau F, Schiffrin EL. Correlation of endothelial function in large and small arteries in human essential hypertension. J Hypertens 2001; 19: 415-20.

7. Endermann D, Pu Q, De Ciucesi C, et al. Persistent remodeling of resistence arteries in type 2 diabetic patients on anti-hypertensive treatment. Hypertension 2004; 43: 399-404.

8. Monnink SH, Van Haelst PL, Van Boven AJ, et al. Endothelial dysfunction in patients with coronary artery disease: a comparison of three frequently reported tests. J Investig Med 2002; 50: 19-24.

9. Perrone-Filardi P, Cuocolo A, Brevetti G et al. Relation of brachial artery flow-mediated vasodilation to significant coronary artery disease in patients with peripheral arterial disease. Am J Cardiol 2005; 96: 1337-41.

10. Pellegrino T, Storto G, Filardi PP et al. Relationship between brachial artery flow-mediated dilation and coronary flow reserve in patients with peripheral artery disease. J Nucl Med 2005; 46: 1997-2002.

11. Landmesser U, Spiekermann S, Dikalov S, et al. Vascular oxidative stress and endothelial dysfunction in patients with chronic heart failure: role of xanthine-oxidase and extracellular superoxidedismutase. Circulation 2002; 106: 3073-8.

12. Costanzo P, Perrone-Filardi P, Vassallo E et al. Does carotid intima-media thickness regression predict reduction of cardiovascular events? A meta-analysis of 41 randomized trials. J Am Coll Cardiol 2010; 56: 2006-20.

13. Halcox JP, Schenke WH, Zalos G, et al. Prognostic value of coronary vascular endothelial dysfunction Circulation 2002; 106: 653-8.

14. Anderson TJ, Uehata A, Gerhard MD, et al. Close relation of endothelial function in the human coronary and peripheral circulations. J Am Coll Cardiol 1995; 26 : 1235-41.

15. Bonetti PO, Pumper GM, Higano ST, Holmes Jr DR, Kuvin JT, Lerman A. Noninvasive identification of patients with early coronary atherosclerosis by assessment of digital reactive hyperemia. J Am Coll Cardiol 2004; 44: 2137-41.

16. Kuvin JT, Patel AR, Sliney KA, et al. Assessment of peripheral vascular endothelial function with finger arterial pulse wave amplitude. Am Heart J 2003; 146: 168-74.

17. Rubinshtein R, Kuvin JT, Soffler M, et al. Assessment of endothelial function by non-invasive peripheral arterial tonometry predicts late cardiovascular adverse events. Eur Heart J 2010; 31: 1142-8.

18. Eckel RH, Wassef M, Chait A, et al. Prevention Conference VI: diabetes and cardiovascular disease: Writing Group II: pathogenesis of atherosclerosis in diabetes. Circulation 2002; 105: e138-43.

19. Balletshofer BM, Rittig K, Enderle MD, et al. Endothelial dysfunction is detectable in young normotensive first-degree relatives of subjects with type 2 diabetes in association with insulin resistance. Circulation 2000; 101: 1780-4.

20. Nitenberg A, Valensi P, Sachs R, Dali M, Aptecar E, Attali JR. Impairment of coronary vascular reserve and Achinduced coronary vasodilation in diabetic patients with angiographically normal coronary arteries and normal left ventricular systolic function. Diabetes 1993; 42: 1017-22.

21. Djaberi R, Roodt J, Schuijf JD, et al. Endothelial dysfunction in diabetic patients with abnormal myocardial perfusion in the absence of epicardial obstructive coronary artery disease. J Nucl Med 2009; 50: 1980-6.

22. Rodbard HW, Blonde L, Braithwaite SS, et al. American Association of Clinical Endocrinologists medical guidelines for clinical practice for the management of diabetes mellitus; AACE Diabetes Mellitus Clinical Practice Guidelines Task Force. Endocr Pract 2007; 13: 1-68.

23. Fox K, Alonso Garcia MA, Ardissino D, et al. ESC Guidelines on the management of stable angina pectoris. Eur Heart J 2006; 27: 1341-81.

24. Bonetti PO, Barness GW, Keelan PC, et al. Enhanced external counterpulsation improves endothelial function in patients with symptomatic coronary artery disease. $J \mathrm{Am}$ Coll Cardiol 2003; 41: 1761-8.

25. Cosentino F, Hishikawa K, Katusic ZS, Lüscher TF. High glucose increases nitric oxide synthase expression and superoxide anion generation in human aortic endothelial cells. Circulation 1997; 96: 25-8.

26. Mäkimattila S, Virkamäki A, Groop PH et al. Chronic hyperglycemia impairs endothelial function and insulin sensitivity via different mechanisms in insulin-dependent diabetes mellitus. Circulation 1996; 94: 1276-82. 